\title{
Espacios de innovación en la relación bilateral Chile-Perú. Los jóvenes como actores emergentes
}

\author{
Diego Riquelme GómeZ*
}

Artículo recibido: 29 de abril de 2020

Artículo aprobado: 8 de febrero de 2021

Doi: https://doi.org/10.12804/revistas.urosario.edu.co/desafios/a.9010

Para citar este artículo: Riquelme Gómez, D. Espacios de innovación en la relación bilateral Chile-Perú. Los jóvenes como actores emergentes. Desafíos, 33(1), 1-36. https://doi. org/10.12804/revistas.urosario.edu.co/desafios/a.9010

\section{Resumen}

Este articulo ${ }^{1}$ aborda la participación de los jóvenes en los Comité de Integración y Desarrollo Fronterizo (cidf.), en el marco de algunas innovaciones en la relación bilateral fronteriza chileno-peruana. Así, se estudia a los jóvenes como actores emergentes en la actividad (para) diplomática interregional entre Arica y Tacna. El objetivo es interpretar etnográficamente la participación de aquellos en estos foros bilaterales, agrupados como Consejo Binacional de la Juventud Tacna-Arica. Se recuperaron y analizaron las trayectorias de estos actores organizados, que convergen junto a otros actores más institucionalizados en el comité. En los resultados, se presenta la caracterización de los jóvenes a partir de las dinámicas de alteridad existentes entre esta pluralidad de actores, en tanto sectores emergentes e invisibilizados en el debate tradicional de las relaciones internacionales.

\footnotetext{
* Universidad Arturo Prat, Iquique, Chile. Correo electrónico: Diegoriquelmegomez@, gmail.com ORCID: https://orcid.org/0000-0003-3476-4140

Resultado del Fondecyt de Iniciación N¹1170568 "Perspectivas y significados en las ciencias sociales chilenas sobre la proyección fronteriza Norte Grande: un análisis relacional desde enfoques predominantes y emergentes".
} 
Palabras clave: etnografía de las relaciones internacionales; paradiplomacia; actores emergentes; comités de integración y desarrollo fronterizo; gubernamentalidad.

\title{
Innovation Spaces in the Chile-Peru Bilateral Relationship. Young People as Emerging Actors
}

\begin{abstract}
This article studies the participation of young people in the Border Integration and Development Committee (CIDF), a novel space in the Chilean-Peruvian bilateral relationship. Youth are studied as emerging actors in inter-regional (para)diplomatic activity between Arica and Tacna. My objective is to ethnographically interpret their participation in the bilateral forums known as the Binational Youth Council of Tacna Arica. The trajectories of these actors are retrieved and analyzed, as is their process of convergence with other more institutionalized actors in bilateral venues. The results characterize youth through the dynamics of otherness amongst a plurality of actors. This analyzes showcases youths as emerging, though usually invisible players in traditional international relations debates.
\end{abstract}

Keywords: Ethnography of international relations; paradiplomacy; emerging actors; border integration and development committees; governmentality.

\section{Espaços de inovação na relação bilateral Chile-Peru. Os jovens como atores emergentes}

\begin{abstract}
Resumo
No marco de algumas inovações na relação bilateral chileno-peruana em suas fronteiras, este artigo aborda a participação de jovens nos Comitê de Integração e Desenvolvimento Fronteiriço (CIDF) como atores emergentes na atividade (para)diplomática inter-regional entre Arica e Tacna. O objetivo é interpretar etnograficamente a participação dos jovens nos fóruns bilaterais, agrupados como Conselho Binacional da Juventude de Tacna Arica. São recuperadas e analisadas as trajetórias desses atores organizados, que convergem com outros atores mais institucionalizados do comitê. Nos resultados, os jovens são caracterizados a partir da dinâmica de alteridade
\end{abstract}


existente entre essa pluralidade de atores, como setores emergentes e invisiveis no debate tradicional das relações internacionais.

Palavras-chave: etnografia das relações internacionais; paradiplomacia; atores emergentes; comitês de integração e desenvolvimento fronteiriço; governamentalidade.

\section{El activismo internacional Arica-Tacna}

Una tarde de febrero del 2014, una comitiva de jóvenes activistas de Arica cruzaba una de las fronteras más transitadas de Latinoamérica. En un ambiente de tensión y expectativa internacional por el Fallo de la Corte Internacional de La Haya por la demanda interpuesta a Chile por parte del Perú, los siete millones de cruces anuales en el paso fronterizo Santa Rosa-Chacalluta parecieron detenerse casi por completo, de no ser por un pequeño grupo de jóvenes que se manifestaba en favor de una ciudadanía por la paz y la hermandad entre ambas naciones.

Estas y otras expresiones de buena vecindad han sido estudiadas durante las últimas décadas en investigaciones sobre las fronteras latinoamericanas, generalmente, asociadas al activismo de gobiernos locales y las sociedades civiles organizadas, que habitan estas zonas fronterizas (González et al., 2016; Oddone, 2018). Se trata de iniciativas de la sociedad civil valoradas por quienes las organizan como espacios para desarrollar valores de buena vecindad y hermandad entre habitantes fronterizos de territorios que son, a la vez, epicentros de conflictos diplomáticos. Se trata del activismo internacional de las regiones, que ha sido estudiado como paradiplomacia transfronteriza (Keating, 2001; Cornago 2016; Oddone, 2018; Bernal, 2018; Astroza, 2018).

En este trabajo, se expone el caso del Consejo Binacional de la Juventud Arica-Tacna (COBIJuv) y su participación en los Comité de Integración y Desarrollo Fronterizo (CIDF). Este es un mecanismo bilateral celebrado todos los años de manera intercalada entre ambas ciudades. Se creó en 1999, cuando por iniciativa conjunta de la Dirección Nacional de Fronteras y Límites en Chile (DIFROL) y su homólogo peruano, la Dirección de Desarrollo e Integración Fronteriza, ambos dependientes de las cancillerías de sus respectivos países acordaron 


\section{4 / Diego Riquelme Gómez}

crear los Comité de Frontera (CF) Arica-Tacna. Estos se concibieron como una instancia en donde se discutirían temas asociados a la gestión y el control fronterizo de ambas ciudades. La agenda de estos foros evolucionó con el tiempo, emergieron nuevos enfoques asociados a la integración y cooperación fronterizas, por lo que en 2012 se decidió reinaugurar estos foros bajo su actual nombre (Pajuelo, 2013; Bernal \& Rochetti, 2017). Esto ha sido clave para la incorporación de actores antes invisibilizados por la diplomacia tradicional. En ellos concurren autoridades de ambos países, funcionarios públicos de ambas ciudades, representantes de cancillería, gobiernos subnacionales y locales y, más recientemente, actores emergentes como los jóvenes agrupados en el coBijuv.

El presente artículo se enfoca en la participación de estos actores a partir del uso de la etnografía como un enfoque emergente en las relaciones internacionales (Neumann, 2012a, 2012b; Cheuk, 2016; Marsden et al., 2016; García Espín, 2017; Montsion, 2018; Kuus, 2019). El objetivo es interpretar etnográficamente la participación de los jóvenes agrupados en el COBIJUV a partir de las experiencias de sus miembros dentro y fuera de los CIDF. Estos hallazgos fueron interpretados a partir del examen que aporta la discusión teórica y metodológica de la etnografía y sus aportes a la disciplina de las relaciones internacionales. Esta discusión puede ser planteada a partir de los siguientes interrogantes que guiaron la investigación: ¿Cuáles son y cómo se desarrollan las prácticas de participación de actores emergentes como el COBIJUV en el trabajo de los CIDF? ¿Cuáles son sus alcances y limitaciones? ¿Qué dinámicas de alteridad hay detrás de ellas? ¿Cuáles son las experiencias de sus miembros? Y, más específicamente, ¿qué rol juegan estos actores en un entramado que, tradicionalmente, es ocupado por otros vinculados a las cancillerías de ambos países?

Una primera hipótesis señala que, dada la presión de diversos actores sociales que se traduce en demandas por participación social en los CIDF, producto de ciertas coyunturas y problemas comunes que afectan a Tacna y Arica, es posible observar cierta apertura en su funcionamiento, al menos en términos discursivos. Esta tendencia 
permite la participación de actores emergentes distintos a los de la diplomacia tradicional en el seno del comité.

En un primer acercamiento, se ha generado un mapa de los actores que convergen en los actuales comités, constatando la diversidad de aquellos en tanto representantes de instituciones y de la sociedad civil (Riquelme, 2020). Esto puede ser interpretado a partir de la pluralidad de actores que convergen en el campo diplomático (Constantinou et al., 2016; Cornago, 2016).

Sin embargo, esta participación tiene ciertas limitaciones o constricciones propias de una lógica tradicional y geopolítica que todavía está presente en la cancillería chilena (Ruiz, 2018). Bajo esta lógica, las regiones fronterizas del Norte Grande son vistas como espacios aislados de las capitales, territorios que el Estado debe resguardar ante su baja densidad poblacional y presencia de riquezas naturales (Mendoza, 2017, p. 120). En particular, la ciudad de Arica, por su ubicación, constituye una avanzada geopolítica, ya que "forma parte de los antiguos sueños irrendentistas de su vecino más próximo, Perú, quien intenta mantenerse en esa área buscando su reclamación futura" (Mendoza, 2017, p. 121).

A partir de esta conjetura se abordan los CF y los CIDF como dispositivos de gubernamentalidad fronteriza; es decir, como una serie de herramientas que permiten la gestión social (Foucault, 2008). En este caso, esta es utilizada en coordinación con un homólogo estatal al otro lado de la frontera. Se plantea la necesidad de profundizar en "las valoraciones que estos actores emergentes tienen de sí mismos y los demás actores” (Riquelme, 2020, p. 50), así como la manera en que se desarrollan y son percibidas las prácticas de participación de los jóvenes en estos comités.

El artículo se estructura de la siguiente manera. En primer lugar, se discute de forma breve la noción de diplomacia ampliada y la pluralidad de actores, en tanto participes de lo que aquí se entiende como un dispositivo de gubernamentalidad en torno a dinámicas de convivencia y control en la frontera. En el siguiente apartado, se discute el uso de 


\section{6 / Diego Riquelme Gómez}

la etnografía en el estudio de las relaciones internacionales (Vrasti, 2008; 2010; Neumann, 2010; García Espín, 2017; Montsion, 2018; Kuus, 2019). Luego, se presentan los alcances frente a los aportes concretos que tiene, para este caso, posicionarme desde una etnografía multisituada (Marcuse, 2001), que evidencie lo que Neumann (2010) entiende como el proceso de socialización del investigador. De esta forma, se propone un enfoque metodológico que puede ser desplegado en el contexto particular del diálogo bilateral y transfronterizo en Arica y Tacna. Después, se describe el contexto geográfico y social en las ciudades de Arica y Tacna, caracterizado por su condición fronteriza, en donde devienen dispositivos como los CF y emerge la participación de actores anteriormente invisibilizados por la actividad diplomática tradicional.

En los resultados se caracterizan los jóvenes a partir de las dinámicas de alteridad que suceden en sus prácticas de participación, en tanto espacio de innovación en la relación bilateral chileno-peruana, por tratarse de actores emergentes o anteriormente invisibilizados en el debate tradicional de las relaciones internacionales.

\section{Entre la diplomacia ampliada y la gubernamentalidad fronteriza}

Para responder a esta problematización algunos enfoques se basan en el reconocimiento de la pluralidad de los actores políticos (Cornago, 2016; Constantinou et al., 2016) que operan en las regiones fronterizas. En el caso de Arica y Tacna, es posible apreciar dinámicas de interdependencia económica y mecanismos de cooperación como los CF (Milet, 2005, Álvarez 2019). Dicho concepto, se sustenta en la diplomacia como heterología, una visión que busca incluir los diversos puntos de vista de los actores que convergen en los espacios diplomáticos y paradiplomáticos, sobre todo fronterizos, al punto que su constitución como sujetos está mutuamente determinada, esta es la calve de la heterología (Cornago, 2016). 
Este concepto es ilustrativo del caso aquí abordado, puesto que emerge "cuando los gobiernos municipales y regionales tienen cuotas significativas de autonomía y vocación transfronteriza, devienen actores destacados de este tipo, pero aquí resulta decisivo el involucramiento de representantes de la sociedad civil" (Cornago, 2016, p. 156). Además, muestra cómo las actividades paradiplomáticas tienden a ser monopolizadas por la cancillería, mediante arreglos institucionales que cooptan la relación internacional entre actores distintos al aparato estatal (Cornago, 2016).

En este corpus teórico, las actividades (para)diplomáticas ocurren de forma cotidiana y tienen inmensas consecuencias en torno a cómo viven las personas en un mundo cada vez más globalizado e interdependiente (Constantinou et al., 2016). Esta es una noción ampliada de la diplomacia, que la entiende como una comunidad que va más allá de los Estados nacionales. Los quehaceres y saberes diplomáticos están, en esta perspectiva, inherentemente inscritos en una sociedad plural y, al mismo tiempo, incrustados en la instrumentalización de la comunicación oficial entre los Estados nacionales (Cornago, 2013, citado en Constantinou et al., 2016, p.5).

Por lo general operan en campos sociales, culturales o semánticos diversos (McConell \& Dittmer, 2016; Oglesby, 2016). Con independencia del lenguaje, las formas y maneras de relacionarse en las actividades diplomáticas están diseñadas para sortear desacuerdos y mantener la conexión e interacción continua en un mundo externo, de por sí plural, que existe independientemente de las representaciones diplomáticas del mismo (Oglesby, 2016, p. 248). En este sentido, McConell y Dittmer (2016) se refieren a culturas diplomáticas ${ }^{2}$ para mostrar cómo estos quehaceres tienden a ser proliferantes y relacionales (p. 109), lo que se relaciona con la idea de ampliación en la diversidad de actores que realizan actividades tanto diplomáticas, como paradiplomáticas. De esta forma, subyace en esta idea la disputa

\footnotetext{
2 Texto original: In this sense, we can see diplomatic cultures, in the plural, as proliferating and relational.
} 


\section{8 / Diego Riquelme Gómez}

por el monopolio de la relación exterior, en este caso, de regiones fronterizas y la diversidad de actores que habita en ellas.

En este punto aparecen los CF como un dispositivo de gubernamentalidad fronteriza, en el que emergen actores antes invisibilizados en el debate y quehacer tradicional de la actividad diplomática. Por dispositivo de gubernamentalidad se entiende un entramado complejo de prácticas, estrategias de gobiernos e instituciones que se cristalizan de forma "calculada y meditada" (Foucualt, 2008, p. 193). En dicho entramado, "el objetivo final es la población" (Foucualt, 2008, p. 63), su protección y control puesto que posibilita la soberanía y gestión del espacio, de manera coordinada entre las cancillerías chilena y peruana para sus territorios fronterizos mediante el dispositivo de los CF y luego de los CIDF.

Bajo esta mirada "la convivencia entre control y protección no es simple contingencia, sino una articulación estructural que caracteriza a las democracias contemporáneas" (Fassin, 2005, citado en Ruiz \& Álvarez Velasco, 2019, p. 695), que en espacios fronterizos tiene ciertas características particulares, las que acá interpreto a partir de la mirada de actores emergentes como los jóvenes en la participación de estos dispositivos. De esta forma, es posible observar que este dispositivo deviene en un espacio que permite el posicionamiento de actores emergentes en su gestión; no obstante, se encuentran ciertas limitaciones institucionales.

Expuestos estos enfoques, es posible apreciar que la participación de estos actores emergentes no es horizontal e invisibiliza las estructuras de poder que median en esta relación. En este contexto, si bien la participación de los jóvenes tiene ciertos alcances y limitaciones, su emergencia permite aventurar la apertura y disputa de un espacio de acción política concreto al incorporar a aquellos actores, que previamente estaban invisibilizados en la diplomacia tradicional. Un enfoque etnográfico en relaciones internacionales permite deconstruir la lógica Estadocéntrica presente en la corriente principal de la disciplina al visibilizar las relaciones de poder existentes entre los actores, conocer las valoraciones, intereses y dimensiones subjetivas 
de los actores investigados, así como la manera en que se configuran para estos las prácticas de participación.

Los jóvenes agrupados en el COBIJUV y sus distintas organizaciones, constituyen un grupo de actores emergentes en la diplomacia tradicional. Pertenecen a un segmento etario pocas veces reconocido por la diplomacia y la política tradicional, en este caso un grupo variado, binacional y con distintas miradas acerca del espacio de los CF y los CIDF, pero también de lo nacional, el desarrollo y la integración. Por ello, es interesante rastrear las narrativas de estos jóvenes, en tanto configuraciones específicas de comprender lo local, lo regional y lo (inter)nacional.

\section{Etnografía y relaciones internacionales}

El uso de la etnografía en relaciones internacionales propone formas de ir más allá en los análisis tradicionales de las relaciones internacionales y se enfoca en la vida cotidiana como un lugar primario desde donde observar la realidad internacional (Lie, 2013, p. 202, citado en Montsion, 2018). Su uso tiene origen en la década de 1990, cuando surgieron una serie estudios de relaciones internacionales que posicionaron a la etnografía como una herramienta rica en términos de datos empíricos, reconociendo cómo las configuraciones institucionales se reflejan en las prácticas concretas de los sujetos (Montsion, 2018). Aunque con matices, este corpus teórico invita a "problematizar el Estadocentrismo al enfocarse en lo cotidiano y en formas de conducir la investigación desde abajo hacia arriba, especialmente en el caso de hechos sociales con un componente transnacional o internacional" (Montsion, 2018, p. 2) 3 $^{3}$ Mi interés no es hacer una revisión exhaustiva de esta literatura, sino mostrar cómo el conocimiento situado de la realidad local permite comprender la manera en que se van tejiendo los complejos entramados en las relaciones de poder, a partir de una

\footnotetext{
3 Traducción propia. Texto original: "problematise state-centrism by focusing on every day and bottom-up ways of conducting research, especially in the case of social facts with a transnational or international component".
} 


\section{0 / Diego Riquelme Gómez}

inmersión reflexiva en el cotidiano, aprehendiendo las estructuras profundas que lo componen.

Sin embargo, el uso de la etnografía como herramienta no necesariamente permite escapar al Estadocentrismo. Para Nina Glick Schiller (2003 citada en Montsion, 2018), esto se vuelve una cuestión sobre cómo las abstracciones utilizadas en los estudios etnográficos son usadas y representadas en la escritura; es decir, los "análisis que igualan a la sociedad con el Estado" sacrifican elementos inductivos del trabajo de campo en la descripción y representación del mundo que elaboran (Glick Schilker, 2003, p. 298, citada en Montsion, 2018, pp. 6-8). De manera que una propuesta de la etnografía en las relaciones internacionales es navegar entre las categorías más abstractas y la experiencia individual del investigador durante el trabajo de campo. Ergo, la atención a las prácticas concretas sobre cómo se hace diplomacia es vital para consolidar este campo.

Otro corpus teórico señala que el "giro práctico" en relaciones internacionales fue influenciado por el trabajo de Pierre Bourdieu y Bruno Latour, quienes señalaron, a comienzos de los 2000, la importancia de establecer un punto de vista desde las acciones cotidianas y el cuestionamiento del sentido común en lo internacional (Montsion, 2018). Un ejemplo de este campo es el trabajo de Kear (2013, citado en Montsion, 2018), quien expuso cómo las actuaciones efectuadas en las protestas del 2011 en el Reino Unido visibilizan debates acerca de leyes de privatización o cómo ciertos discursos políticos y protestas reproducen espacios internacionales, haciendo énfasis en cómo podemos tener un entendimiento más completo de las prácticas de las relaciones internacionales. En esta misma línea, el estudio de Paul Dwyer (2013, citado en Montsion, 2018) documenta y explica el rol de Australia en la guerra civil de Bougainville, a través de un proyecto teatral en donde mezcla la autoetnografía con la autobiografía para contar su experiencia de vida en esa ciudad, revisitando desde allí la memoria colectiva de ese conflicto internacional.

Por otra parte, Merje Kuus (2019) resalta cómo la vida cotidiana se relaciona con las instituciones que diseñan las políticas, señalando la 
importancia del contexto y la experiencia sensorial en el trabajo académico. En su argumento, resalta cómo las prácticas diplomáticas y burocráticas pueden ser leídas a partir de la puesta en escena con que se desarrollan. Es decir, la manera en que las prácticas diplomáticas son normadas por ciertos protocolos y la experticia en la negociación, junto con otras que se enseñan en las academias diplomáticas. En esta lectura, el conocimiento diplomático es un conocimiento práctico. El propósito de estos actores tradicionalmente ha sido la canalización del interés nacional en lugares específicos hacia el extranjero; su experticia es necesaria para la toma de decisiones basadas en una visión de alguien que efectivamente se encuentra en ese lugar (Kuus, 2018, p. 7). Es posible comparar el oficio del diplomático con el del antropólogo, puesto que resume la máxima malinowskiana de "estar allí" para comprender y situar realidades concretas de manera localizada, absorbiendo los "imponderables de la vida cotidiana" (Malinowski, 2001).

En esta línea, Kuus (2019) señala que los obstáculos metodológicos para la etnografía general son barreras sociales, lo que lleva a los investigadores a despreciar actividades humildes y fáciles, pero fértiles, en favor de ejercicios difíciles y estériles analíticamente hablando (Bourdieu, 1984, citado en Kuus, 2019, p. 10). De esta forma, es común en los investigadores convencionales querer vincular inmediatamente las especificidades encontradas en el trabajo de campo a principios generales abstractos, como si estudiar las particularidades fuese insuficientemente analítico o poco riguroso. Así, este autor propone dejar nuestras investigaciones abiertas hacia la ambigüedad, la contingencia y la especificidad local de las prácticas burocráticas (2019, p. 10), teniendo claro que siempre habrá "puntos ciegos" en todo posicionamiento.

Otros autores mencionan el estudio de la diplomacia cotidiana — everyday diplomacy - como un campo en desarrollo que involucra la geopolítica crítica y la geografía política, enfocadas en el rol que la cultura juega en las prácticas diplomáticas (Mardsen et al., 2016). Más específicamente, señalan que el auge del constructivismo y el foco en el discurso, paradójicamente, resultaron en la infravaloración del rol que juegan las ideas 
en la diplomacia y en la construcción de representaciones colectivas e individuales (Mardsen et al., 2016, p. 3). Frente a esto, Neumann (2010) propone prestar atención a las formas de intercambio que suceden en los "lugares diplomáticos", en una etnografía sobre las prácticas de interacción y comunicación institucionalizadas en "tiempos, lugares y formatos de reunión, mutuamente reconocidos" (Neumann 2012a, p. 5; Neumann 2012b, pp. 15-44, citado en Mardsen et al., 2016, p.14) ${ }^{4}$.

En suma, los antropólogos clásicamente se han cuestionado los orígenes de la sociedad humana y la cultura a partir de sus dinámicas de intercambio e interacción. Estas actividades son inherentes al quehacer diplomático. De acuerdo a Geertz (1991), la cultura puede ser entendida como un entramado complejo de textos, una telaraña de significados que las mismas personas han tejido y que los antropólogos intentan desenmarañar para comprender las estructuras profundas que dan sentido a las prácticas cotidianas. En este sentido, Vrasti $(2008 ; 2010)$ propone que la etnografía no se trata solo de un método, sino que, cuando es aplicada con rigurosidad, permite desafiar las abstracciones que la disciplina de las relaciones internacionales ha construido tradicionalmente.

\section{Metodología y hallazgos}

Para responder a las preguntas planteadas en este artículo, realicé una etnografía multilocal (Marcus, 2001) entre Arica, Tacna, Santiago y Lima, apoyándome en la discusión del uso de la etnografía como herramienta novedosa en los estudios de las relaciones internacionales (García Espín, 2017; Montsion, 2018). Entiendo la etnografía multilocal como un proceso reflexivo de recogida y análisis de datos que se caracteriza por "examinar la circulación de significados, objetos e identidades culturales en un tiempo-espacio difuso" (Marcus, 2001,

\footnotetext{
4 Traducción propia. Texto original: Neumann proposes to explore such forms of exchange by paying attention to the 'diplomatic sites' at which diplomacy actually does take place, and the ethnography of institutionalized practices of interacting and communicating in 'mutually recognised times, places, and formats for meetings'.
} 
p. 111), en este caso, a distintas escalas, pues considero dicha circulación entre Arica y Tacna, pero también entre las respectivas capitales nacionales, pues es desde allí que se piensan dispositivos como los CF.

Durante la estadía en Lima, pude entrevistarme con altos funcionarios de la cancillería peruana. ${ }^{5}$ En Santiago hice lo mismo con los homólogos de la cancillería chilena. Esto me permitió conocer la perspectiva institucional y la forma en que los centros y tomadores de decisiones piensan y diseñan estas políticas públicas. En mayor profundidad, me entrevisté con jóvenes de Arica y Tacna pertenecientes a diversas organizaciones que convergen en el COBIJUV. En dichas conversaciones pude aprender la trayectoria tanto de sus miembros, como del consejo, así lo llaman coloquialmente sus miembros. Por su parte, la estadía prolongada durante el trabajo de campo en Arica, Tacna, Lima y Santiago hizo posible contextualizar los lugares y la circulación de saberes y prácticas entre aquellos.

Como expuso Michael Shapiro (2013, citado en Montsion, 2018, p. 10), los estudios etnográficos en relaciones internacionales permiten poner en el centro las representaciones y percepciones desde la diversidad de los actores involucrados, al indagar cómo "poblaciones específicas ven el mundo, y cómo su realidad está integrada de manera única en el mundo" (Montsion, 2018 , p. 18). ${ }^{6}$ En esta línea, Neumann (2010), después de un detenido trabajo etnográfico sobre las rutinas diplomáticas en distintas latitudes, mostró que cualquier investigación que trate sobre las prácticas diplomáticas y los discursos debería considerar la multiplicidad de intervenciones e interacciones subestatales y de la sociedad civil en el ámbito diplomático. Esta multiplicidad la interpreto a partir de la noción de pluralismo diplomático de Cornago (2016), la cual se entiende como la diversidad

\footnotetext{
5 Información recabada en el marco de mi pasantía de investigación financiada por el concurso Integración y Buena Vecindad Chile-Perú, Perú-Chile 2019, Fundación Konrad Adenauer.

6 Traducción propia. Texto original: "how specific populations see the world and how their reality is uniquely integrated into the world".
} 
de actores que participan en estas instancias de diálogo interestatal e interregional.

La antropología permite estudiar a las entidades políticas o Estados a través de los efectos que tienen en la vida cotidiana (Neumann, 2010; Montsion, 2018). En particular, permite explorar las posibles implicaciones del pluralismo social, la complejidad doméstica y las continuidades territoriales en las dinámicas institucionales y políticas que pueden tanto facilitar como complicar la formación de relaciones diplomáticas pacíficas y duraderas entre Estados.

En este sentido, la etnografía permite revelar las importantes implicaciones de ese pluralismo social para la diplomacia chileno-peruana. El método etnográfico se basa en un acercamiento inductivo-deductivo, puesto que se analizan al mismo tiempo múltiples realidades de manera simultánea (Durand, 2014). En esta línea, tomando como premisa el hecho de que las metodologías demasiado deductivas tienden al Estadocentrismo, Jean Michel Montsion (2018) plantea que "hay lecciones que pueden extraerse de cómo la antropología social ha tratado de abordar esto, incluyendo cómo estudiar los Estados o entidades políticas a través de sus efectos en la vida cotidiana, las prácticas materiales, etc.” (Montsion, 2018, p. 7). ${ }^{7}$

De esta forma, la etnografía remite al compromiso de incorporar representaciones de la vida cotidiana en el estudio de la política internacional y vecinal, que requiere del compromiso ético y político de alejarse de la mirada dominante Estadocentrista para comprometerse directamente con las experiencias internacionales de los grupos humanos en sus propios términos, en relación con los procesos estructurales en los que se inscriben (Montsion, 2018, p. 8). Esta idea se ajusta con la de pluralismo diplomático presente en la franja fronteriza tacno-arqueña, donde operan distintos actores que reivindican una actividad diplomática:

\footnotetext{
7 Traducción propia. Texto original en inglés: "However, there are lessons that can be gathered from how social anthropology has attempted to address this, including how to study states or political entities through their effects on everyday life, material practices and so forth."
} 
sociedad civil, agentes del Estado y gobiernos locales y regionales, entre otros, unas veces en pugna, otras en colaboración.

Como ejercicio de recolección y análisis de información etnográfica, tomé la noción acerca del proceso de socialización del investigador propuesta por Neuman (2010) para presentar enfoques alternativos en relaciones internacionales. Esto me permitió trazar la manera en que se mueven e instalan ciertos discursos, así como sistematizar las impresiones que aparecen en la socialización con los interlocutores de esta investigación: jóvenes activistas en la relación internacional de Arica y Tacna.

Se trató de un ejercicio metodológico artesanal, cercano al aprendizaje de un oficio (Durand, 2018): el de activistas trasfronterizos que hacen converger el interés de los jóvenes a nivel binacional. La apuesta esgrimida aquí implicó una aproximación empírica a lo que Wacquant (2015) identifica en la sociología de Bourdieu como "el suelo de las realmente existentes conductas y significaciones políticas (...captando] las capacidades políticas inscritas en estructuras formales[, así como] las diversas inclinaciones y expectativas de actores concretos [en el] juego democrático" (p. 15). De esta manera, abordé las prácticas y valoraciones de estos actores en el comité, mencionando sus estrategias de legitimación.

Por último, el acento en la multiplicidad de espacios permite comprender las conexiones entre territorios distintos al salir de "los lugares y situaciones locales de la investigación etnográfica convencional al examinar la circulación de significados, objetos e identidades culturales en un tiempo-espacio difuso" (Marcuse, 2001, p. 111). En este sentido, estudiar las prácticas que configuran el corredor transfronterizo Arica-Tacna, desde una perspectiva etnográfica, permitiría contrastar e interpretar el contenido de relaciones paradiplomáticas Tacna-Arica, desde la experiencia de los sujetos, situada localmente, pero también multisituada en las dinámicas de alteridad; es decir, de percepción mutua entre los actores tanto a nivel regional como nacional. 


\section{Entre aquí y allá: Actores emergentes en la dinámica transfronteriza Arica-Tacna}

Arica y Tacna son dos ciudades geográficamente contiguas. Ambas están situadas en el extremo norte de Chile y el extremo sur del Perú, respectivamente, lo que las convierte en ciudades fronterizas. Una de sus características es la compleja dinámica transfronteriza que las conecta y las separa al mismo tiempo. Diversos autores concuerdan en que la relación bilateral entre Chile y Perú ha estado marcada por rezagos del pasado que provienen de la Guerra del Pacífico, los acuerdos del Tratado de Lima de 1929 y, más contemporáneamente, por la disputa marítima tratada en La Haya (Ovando \& González, 2018). Sin embargo, a pesar de este contexto de disputa territorial entre ambos países, las ciudades de Arica y Tacna han establecido intensas relaciones transfronterizas, donde una de estas expresiones son los CIDF.

En 2019 se realizó el VIII CIDF Arica-Tacna, instancia que cumplió 20 años desde su concepción original como CF. Se trata de un foro bilateral que se ha celebrado anualmente desde 1999, de manera intercalada entre las ciudades de Arica y Tacna. Entonces llamados CF, tras 12 años de funcionamiento se reestructuraron bajo su actual nombre de CIDF, en el 2012, debido a que ambas cancillerías buscaron agregar el elemento de la integración y el desarrollo fronterizos en estos foros bilaterales. Otra de las razones fue la baja en la participación y el porcentaje de cumplimiento en los acuerdos tomados entre el 2000 y el 2011 (Pajuelo, 2013; Bernal \& Rochetti, 2017; Alvarez, 2019).

La creación de este dispositivo en la década de 1990 surgió en el contexto latinoamericano de redemocratización del continente tras el periodo de dictaduras, que permitió la emergencia de una nueva agenda de integración regional y la reapertura de los canales diplomáticos en los países de la región (Cornago, 2016). Sin embargo, su creación se puede rastrear a la década de 1980 entre el Estado chileno y sus países vecinos, a partir de una iniciativa de la Dirección Nacional de Fronteras y Límites de Chile (DIFROL), un órgano de la Cancillería encargado de resolver asuntos limítrofes y de tránsito 
en estas zonas. Así, en una primera instancia los comités tenían el objetivo de "resolver problemas operativos del tránsito y tráfico (...) así como la cooperación e integración regional, y considerar e impulsar proyectos conducentes al desarrollo y mejor entendimiento entre las poblaciones fronterizas" (DIFROL, citado en Álvarez, 2019, p. 56). El primero de ellos se realizó en 1987 con Argentina, diez años más tarde, en 1997, se creó uno con Bolivia (actualmente inactivo) para las regiones de Tarapacá y Antofagasta por el lado de Chile y los departamentos de La Paz, Potosí y Oruro por el lado boliviano. Luego, en 1990 se creó el Comité de Frontera Chile-Perú, que comprendía la actual región de Arica y Parinacota en Chile y el Departamento de Tacna en el Perú (DIFROL, citado en Álvarez, 2019, p. 56). Actualmente, existen alrededor de 16 de estos dispositivos a lo largo de Chile; no obstante, aquí me refiero solo al caso de Chile-Perú, por ser una realidad más próxima, así como uno de los más activos según quienes participan en este.

Así, el Comité de Frontera Chile-Perú nació con los objetivos de abordar problemas de tráfico de personas, vehículos y bienes; promover la cooperación e integración regional, e impulsar proyectos orientados al desarrollo de las poblaciones fronterizas (Pajuelo, 2013; Cornago, 2016; Bernal \& Rochetti, 2017; Álvarez 2019). Uno de sus acuerdos más emblemáticos fue la creación del Complejo Integrado Santa Rosa Chacalluta, que se expresó en flujos más eficientes y expeditos, alcanzando 7 millones de cruces anuales hacia un lado y otro de la frontera (Dilla \& Álvarez, 2019).

Tras 12 años de funcionamiento, las cancillerías de ambos países decidieron cambiar el nombre de los CF a CIDF. Su estructura de funcionamiento consta de una coordinación general, formada por altos funcionarios de las cancillerías de ambos países, quienes intercalan la presidencia cada año. Luego se encuentran las tres comisiones principales: Facilitación Fronteriza; Infraestructura, Transporte y Normas, e Integración. Cada una se separa en diversas subcomisiones, que abordan temas y acuerdos competentes a los órganos involucrados. Esta estructura principal de funcionamiento no ha cambiado desde entonces; sin embargo, con el paso de los años han aparecido nuevas 
subcomisiones como la de Voluntariado Juvenil, en donde participan los miembros del COBIJuv.

Al respeto, Álvarez (2019) argumenta que durante los primeros años el comité formaliza vínculos que en términos prácticos ya existían entre algunos vecinos, autoridades y gremios locales. Se trataría de "un espacio político-social de concertación transfronteriza en el que el mundo social ve la oportunidad de transformar y acercar la alta política exterior de los Estados nacionales al territorio fronterizo" (p. 64). En este sentido, a partir de la literatura revisada respecto de los comités (Pajuelo, 2013; Ovando, 2017 Bernal \& Rochetti, 2017; Álvarez, 2019) se puede inferir que se trata de una novedad institucional en la relación trasfronteriza entre ambos países.

En este contexto, la incorporación de los jóvenes en la agenda de este mecanismo ha permitido "traer nuevos aires" al comité según altos miembros de ambas cancillerías. Cabe añadir que los encuentros con estos funcionarios están marcados por protocolos que en algunos casos incluyeron la negativa a grabar las conversaciones. Las observaciones e interacciones en los lugares diplomáticos (Neumann 2012a; Neumann 2012b) no son objeto del análisis aquí presentado, ergo, dicho acercamiento al terruño de la práctica política (Kuus, 2019) permitió contextualizar el activismo internacional de los jóvenes como espacio de innovación en lugares que tradicionalmente son cerrados. El hermetismo sigue caracterizando a la alta política, y pese a que la emergencia de los jóvenes puede ser leída como una innovación institucional, esta tiene ciertos alcances y limitaciones, objeto de este artículo.

Hecha esta discusión, a partir de las trayectorias de sus miembros se identificaron e interpretaron cuales y cómo se desarrollan las prácticas de participación de los jóvenes pertenecientes al COBIJUV, en particular a partir del trabajo que realizan como parte de los CIDF. En el relato se aborda la génesis del consejo, los alcances y limitaciones de esta participación, la valoración que hay de estas prácticas y, más específicamente, el rol que estos actores desempeñan en un entramado que ha sido tradicionalmente ocupado por las cancillerías y 
gobiernos locales de ambos países, en el que solo recientemente se ha incorporado la sociedad civil. Con ello busco presentar a los jóvenes como actores emergentes en el activismo transfronterizo, que ocupa espacios de innovación política y diplomática.

\section{Cruzamos cuando no había nadie}

El COBIJUV es una agrupación que busca "ser un ente que trabaje por la integración y la paz entre las dos naciones, propiciando la participación de la juventud en el desarrollo político, social, económico y cultural de nuestros países" (COBIJuv, s. f.). Sus orígenes se remontan al 2010, cuando a raíz del terremoto en Chile, agrupaciones de voluntariados juveniles en Tacna se organizaron para realizar actividades benéficas en favor de familias afectadas, abriendo una alianza estratégica con el Consulado General de Chile en Tacna. Dicha relación sentó las bases para que luego, en 2011, se hiciera el primer encuentro de jóvenes de Perú y Chile, provenientes de organizaciones de voluntariado juvenil tanto de Arica como de Tacna (Diario El Correo, 2016).

En el 2014, realizó uno de sus primeros pronunciamientos en el marco de la disputa marítima entre Perú y Chile ante la Corte Internacional de La Haya. En esa ocasión, los jóvenes llamaron a la calma y la hermandad ante incipientes, aunque preocupantes voces nacionalistas que resonaron local y nacionalmente, mediante una actividad en la que se tomaron de las manos en la Casa de la Cultura de Arica, que posteriormente fue replicada en Tacna. El 29 de enero de ese año, estos jóvenes dirigentes de Arica y Tacna crearon el COBIJuv "para reforzar los lazos de hermandad entre ambas ciudades" (Carrasco, 2014). Desde entonces, el COBIJUV ha evolucionado, han ingresado nuevas organizaciones y se han ido otras; a la fecha es posible observar al menos un recambio generacional, pues sus miembros fundadores ya han dejado la organización.

El acto simbólico que realizaron para el llamamiento a la hermandad es visto por sus adherentes como uno de sus hitos constituyentes. Uno de sus miembros fundadores recuerda que un jueves, 23 de enero del 2014, se pusieron de acuerdo a través de redes sociales para 


\section{0 / Diego Riquelme Gómez}

pronunciarse ante la tensión territorial que se respiraba tanto nacional como localmente. Un día en el que los siete millones de cruces anuales cesaron casi por completo, de no ser por el paso decidido de estos jóvenes: "La idea era hacer un pronunciamiento aquí y allá, porque queríamos llamar a la calma y a la hermandad. Era un clima beligerante... el día en que viajamos para Tacna no había ni un alma en la frontera" (Miembro fundador 1 en entrevista en Arica, agosto de 2019).

Es así como activistas de diferentes organizaciones se movieron entre aquí y allá, en un acto de pronunciamiento. Previamente a que llegara la delegación chilena a Tacna, uno de los fundadores peruanos solicitó apoyo en el consulado chileno de la ciudad. La respuesta fue inmediata y mediática.

Nos prestó toda la ayuda, nos respondió inmediatamente, se consiguió un hotel y citó a la prensa. Allí pusieron una mesa y nos dijo como colocar las banderas, y de qué manera distribuirnos para dar el comunicado (Miembro fundador 2, en entrevista en Tacna, julio del 2019). ${ }^{8}$

Tiempo después, fueron invitados por este mismo cónsul a participar en el CIDF, específicamente como una mesa dentro de la subcomisión de Género, lo que sentó un nuevo precedente en la trayectoria de la organización. Fruto de esta participación, los jóvenes convocados comenzaron a incorporar el elemento binacional en torno a actividades propias de cada organización pertenecientes al CoBIJuv, que se fueron ampliando con los años.

En conversación con algunos de sus miembros actuales, así como con funcionarios de ambas cancillerías, me comentaron en varias ocasiones que esta alianza estratégica con el consulado chileno en Tacna, en primer lugar, no hubiese sido posible sin el apoyo del aquel entonces cónsul general de Chile en Tacna, quien conocía personalmente a los

8 Nótese el uso de convenciones propias de la actividad consular. 
jóvenes que llevaron a cabo estas iniciativas. Ellos fueron los fundadores del CoBIJuv. Luego, hicieron uso de sus redes como activistas y voluntarios para convocar a otros jóvenes a que participaran.

Uno de los requisitos de ingreso - hasta hoy- es pertenecer a alguna agrupación de voluntariado o activismo juvenil. Es decir, el COBIJUV está formado por un conglomerado de agrupaciones de jóvenes desde las cuales se envía un representante para participar en las sesiones. Otro, igual de importante, es que no pueden pertenecer a partidos políticos. De hecho, es por esta razón que sus miembros fundadores han dado un paso al costado, pues uno de ellos hoy es un activo concejal en Arica.

Una miembro, representante de la organización Tacna Valley, comentó que pueden ingresar jóvenes entre los 15 y 30 años, siempre como representantes de organizaciones que trabajen en actividades hechas por o para jóvenes: "Se trabaja en 5 ejes: ciudadanía, medio ambiente, emprendimiento e innovación social, arte y cultura binacional" (Entrevista en Tacna, agosto 2019). Por ejemplo, la organización a la que pertenece esta joven, que forma parte del COBIJuv, difunde el emprendimiento entre jóvenes a través de capacitaciones en proyectos de gestión empresarial. En este caso, ella ingresó al cOBIJuv a través de una invitación a un directivo de su agrupación.

Cuando ingresamos, el entonces director de Tacna Valley, era amigo de los jóvenes del consejo binacional, quien les comenta un poco del trabajo que hacemos y nos piden que ingresemos a través del eje de emprendimiento que se había incluido recientemente en el consejo, luego él se retira y me asignan a mí para trabajar con el consejo binacional (Representante de Tacna Valley, entrevista en Tacna, agosto 2019).

El caso de la Red Universitaria Ambiental (RUA) de Tacna es similar. Se trata de una red peruana con nodos a nivel nacional desde Iquitos hasta Tacna, que ingresa como una de las primeras organizaciones encargadas del medioambiente. Fueron incorporados formalmente 


\section{2 / Diego RiQuelme Gómez}

en 2018, tras presentarse en 2017, en el Encuentro Internacional de Jóvenes Líderes (EIJOL), que para entonces se había consolidado como una instancia de congregación anual desde donde presentar avances y generar programas. Allí se invita a agrupaciones externas para que muestren el trabajo propio y se postulen en base a una evaluación hecha por comitiva externa convocada por el COBIJUV. Este fue el mecanismo mediante el cual esta red ingresó.

Una pregunta que hice a varios miembros del COBIJUV fue cómo se puede pertenecer a este. La respuesta siempre fue que debía pertenecer a alguna organización de jóvenes voluntarios de Arica o Tacna. La única distinta fue la del representante de World Industrial, una agrupación que promueve el Hip-Hop en las poblaciones, con el objetivo de alejar a los niños de situaciones de vulnerabilidad, mediante talleres sobre las diversas disciplinas de este arte urbano.

Bueno con esta misma entrevista ya te estás acercando un poco, pero sería bueno por ejemplo que tu como antropólogo te pudieras juntar con otros antropólogos o investigadores interesados en investigar este tema, en hacer tratados de paz haciendo investigaciones que nos pueda servir a nuestro trabajo, sería bonito por ejemplo que juntaras investigadores de Perú con un grupo de Chile, sería bacán (sic) (Representante de World Industrial, entrevista en Arica, agosto 2019).

La familiaridad con que elaboró esta respuesta puede estar relacionada con el ánimo principal del comité: la convergencia de los intereses de los jóvenes de manera binacional. El interés por el activismo internacional es valorado positivamente por este dirigente y el espacio académico es visto como lugar posible desde donde posicionar este activismo. Como mostraré, en el campo en que opera el COBIJUV, se juegan alteridades a partir del reconocimiento mutuo entre las institucionalidades y los agentes que las componen y las relaciones de poder que las configuran. 


\section{Siempre hubo que pelear el reconocimiento}

Uno de sus miembros perteneciente a la Asociación de Jóvenes Artistas de Arica resume cómo la organización fue adquiriendo su carácter a partir del activismo tanto dentro como fuera del CIDF:

Nos juntábamos de manera separada en cada ciudad, cada uno en Tacna o en Arica, pero manteníamos contacto por redes sociales. El consejo empezó a operar como una red de voluntariado (...) había actividades que partían como iniciativa propia de cada organización, quienes le pedían ayuda al consejo y se reformulaba la actividad dándole el giro binacional de integración (Miembro AJA, entrevista abril, 2020).

Tal como señala este miembro, el COBIJUV comienza a funcionar como una red en la que convergen las diferentes organizaciones en la órbita de este. Cuando una organización plantea alguna iniciativa, echa mano de los contactos transfronterizos y binacionales que posee. En este sentido, el COBIJUV si bien surge bajo el alero de la institucionalidad investida por cancillería, rápidamente la desborda toda vez que sus miembros orbitan dentro de otros campos de acción política que se superponen y son homólogos en el activismo internacional, normado o no. "Generalmente las actividades se proponían en el comité, sin embargo (...) con el tiempo el consejo fue adquiriendo cierta autonomía y, esto se fue definiendo mejor cuando tomó más protagonismo en el comité" (Miembro de AJA, entrevista abril, 2020).

Pero este protagonismo tuvo limitaciones. Según algunos miembros, había reticencias provenientes de ciertos miembros de la cancillería y otros actores que participaban de los comités para reconocerlos como una subcomisión. De esta forma, en un principio, el COBIJUV participó como una mesa dentro de otra subcomisión, y solo después de tres o cuatro años de participar, la Coordinación General del CIDF los reconoció como la subcomisión de voluntariado juvenil.

Siempre hubo que pelear el asunto del reconocimiento dentro del mismo comité. Trabajamos durante todo el año para hacer la 
propuesta en el comité para que nos reconocieran como subcomisión de Voluntariado Juvenil. Los dos fundadores, quien eran las caras más visibles (...) se presentaron ante la Coordinación General del Comité, y luego se aprobó la constitución de aquella (Miembro de AJA. Abril. 2020).

La formalización se materializó mediante un documento oficial emitido por la Coordinación General del CIDF, que es reconocido por ambas cancillerías. En la reflexión que este miembro hace, el COBIJuv va más allá del plano institucional y se inscribe en un sentimiento de identidad colectiva en torno a la fraternidad entre las sociedades regionales de frontera, el cual tiene su hito fundacional en el primer pronunciamiento realizado hace seis años:

Recuerdo la sensación que había, de defender nuestra postura y demostrar confianza, entregamos el apoyo y suerte a los fundadores para que todo saliera bien, y finalmente la alegría y celebración una vez recibida la noticia, que en cierta medida era entrar a un nivel de política más alta que antes no teníamos (Miembro de AJA, abril, 2020).

El reconocimiento por parte de los órganos estatales de política exterior es visto como un avance por estos agentes. De esta forma, el 4 de septiembre del 2014 sesionó por primera vez el Grupo de Voluntariado Juvenil dentro de la Comisión de Integración (CIDF, 2014). En el documento que oficia su conformación, se señalan las actividades realizadas durante ese año como el Pronunciamiento de La Juventud de Paz e Integración por el Conflicto de la Haya, actividades artísticas, de concientización ambiental y de voluntariado en hospitales, entre otras.

Una inferencia interesante respecto de la arquitectura que conforma esta organización, es que, si bien no se admiten agrupaciones vinculadas a partidos, existe la percepción del consejo como espacio político. Un ejemplo es la percepción de la apertura y apropiación de este nuevo espacio institucional en el foro bilateral como un logro importante 
por los miembros del COBIJUV, que permite consolidar una autonomía relativa respecto de este y las cancillerías al ser reconocido como subcomisión. Dicho reconocimiento escaló hasta la más alta esfera de representación diplomática. En el II Gabinete Binacional ChilePerú ${ }^{9}$ (Ministerio de Relaciones Exteriores, 2018), los presidentes de ambos países lo incorporaron en el documento oficial de la reunión lo que puede ser interpretado como un acto de reconocimiento y, al mismo tiempo, como una estrategia de coaptación de las actividades paradiplomáticas por parte de la cancillería.

Una referencia que tuvo resonancia en varios miembros de ambas cancillerías, así como en otros actores locales, fue que la inclusión de los jóvenes pudo "dar nuevos aires" al CIDF. Ejemplo evidente de ello es el hecho de que la invitación por el entonces cónsul general de Chile en Tacna para que participaran coincide con el cambio de nombre de este dispositivo. En un primer momento, la participación de los jóvenes se logra mediante un arreglo institucional. Sin embargo, al ir adquiriendo cierta autonomía relativa respecto de las cancillerías, el CIDF va tomando su carácter propio. Esto es posible de observar cuando la participación e inclusión de otros actores se hace mediante relaciones de confianza, previamente establecidas a través de otras redes distintas a este, pero que convergen allí.

El COBIJUV es visto por las otras subcomisiones como una de las más activas, sobre todo porque es la única que no cuenta con un financiamiento fijo a diferencia de las otras que tienen sus propios fondos, al provenir de aparatos públicos.

Somos los regalones de ese comité, todas las personas nos admiran por todo lo que hacemos sin dinero, y por eso muchos de estos diplomáticos están al tanto de nuestro trabajo, quieren trabajar con nosotros y contactarnos (Miembro de World Industrial).

\footnotetext{
9 Instancia diplomática postfallo de La Haya a más alto nivel que, dentro de sus cinco ejes, tiene una agenda de Desarrollo e Integración Fronteriza.
} 


\section{6 / Diego Riquelme Gómez}

Porque todas las subcomisiones que integran el comité son entes del Estado. Ninguna es privada a excepción de la de la sociedad civil (...) la única que es privada que no tiene financiamiento exclusivo, es la de voluntariado juvenil. Porque las entidades que participan son del Estado, entonces cada una tiene su financiamiento. En nuestro caso no, todas somos organizaciones sociales que no pertenecen al estado (Miembro de Tacna Valley, entrevista en Tacna, agosto 2019).

El financiamiento para el EIJOL proviene de un fondo interno del consulado chileno en Tacna. Fuera de este no tienen otras fuentes directas. En el CIDF del 2019 solicitaron "más apoyo en financiamiento para ambas partes tanto Arica como Tacna, porque solamente el EIJOL es el que tiene presupuesto establecido" (Miembro de RUA, entrevista en Tacna, agosto 2019). Es por esto que los integrantes del COBIJUV echan mano de los recursos, redes y contactos de sus propias agrupaciones para poder ejecutar las actividades que realizan durante el año. Otra limitante es la amplitud de actividades que realizan y la necesidad de que resuenen aquí y allá.

Entonces era como que nos recargábamos mucho, de muchas actividades, si bien todos eran a través de gestión con entidad privada, a veces el consulado nos financiaba sólo una actividad que siempre ha sido el EIJOL, de ahí para otras actividades no hay un presupuesto específico (Miembro de Tacna Valley, entrevista en Tacna, agosto 2019).

Respecto de las posibles maneras de sortear la limitante económica señalaron que se encuentran "avanzando hacia el financiamiento de Cooperación Internacional” (Miembro de RUA), que según me explicaron es un fondo que gestionan las agencias de cooperación internacional de Chile y Perú. Dicha entidad bilateral forma parte de la misión diplomática chilena en el Perú y posee una serie de fondos y becas concursables focalizados en diversas áreas (Ministerio de Relaciones Exteriores, s. f.). Desconozco a la fecha el avance de esto; sin embargo, las limitaciones no son solo económicas. 
Otra limitante que identificaron los miembros fue lo difícil que era mantener una directiva centralizada que organizara a las agrupaciones tanto en Arica como en Tacna. Es por esto que en la reunión que sostuvieron en el comité del 2019, decidieron elegir una directiva por ciudad.

Había necesidad de una junta directiva aquí y allá poder revisar mejor las cosas y nunca había pasado eso aquí en Tacna respecto al consejo binacional, entonces es propio y les pareció bien y se dio a la votación de manera incógnita secreta, bastante simbólico y bastante transparente (Miembro de RUA, entrevista, agosto 2019).

La presencia de dos directivas aquí y allá refuerza la idea del COBIJUV como una red fronteriza, pero que se adapta según las particularidades de cada ciudad. Mientras que en Tacna actualmente es posible observar cinco o seis agrupaciones activas que orbitan en este, en el caso de Arica son solo dos o tres, por lo que hay, en términos absolutos, mayor participación de un lado que del otro. Sin embargo, esto no es excluyente para la realización de sus actividades y por lo que decidieron comenzar a enfocarse en menos ejes temáticos por año. En tanto, la posibilidad de reunirse de manera presencial en el CIDF, permite resolver esta y otras cuestiones operativas:

Pudimos establecer las deficiencias que teníamos e hicimos una suerte de sinceramiento, porque había varias cosas que eran difíciles de expresar y todo fluyó, se pudieron ver cuáles eran las debilidades que tenía Arica (...) fue una retroalimentación bastante buena, porque es muy difícil ponernos de acuerdo, hay muy pocas ocasiones a veces por ejemplo cuando hacemos el estatuto lo hacemos vía web, pero es diferente cuando es en persona y están las organizaciones (Miembro de RUA).

La dificultad que menciona este miembro, permite comprender que el COBIJUV goza de cierta autonomía respecto del CIDF, el cual es visto como un espacio para poder resolver cuestiones operativas en persona, lo que tiene una valoración distinta a cuando las coordinaciones no 


\section{8 / Diego Riquelme Gómez}

son presenciales. El CIDF permite el uso de su arquitectura al reconocer a los jóvenes como subcomisión, y también permite consolidar en el tiempo lazos fraternales: "Las veces que había comité después nos íbamos a la casa de uno de los compañeros y compartíamos con los compañeros de Tacna, nos quedábamos en las casas de los compañeros" (Miembro de AJA).

La institucionalidad se trasciende en la camaradería. El alojamiento en el hogar de un colega es una práctica que trasciende la esfera pública y se instala en lo privado, fuera de cánones diplomáticos y los convencionalismos propios del CIDF.

En tanto, los jóvenes, reconocen una relación jerárquica, respecto de cancillería, pero con matices: "Estamos en el último eslabón, pero al final no es tanto...o sea, nosotros podemos articular con las otras subcomisiones si es que lo deseáramos, o sea hay espacios libres para que las otras subcomisiones se pueden entrelazar y articular" (Miembro de Tacna Valley).

De forma que existe espacio para plantear inquietudes y agendas nuevas, pero es limitado: "Claro nos dan espacio, pero bueno, desde abajo como observación podemos plantear añadiduras que necesitamos algunas cosas que quizás nos incomoden, molesten o queremos mejorar qué se yo, como te comentaba uno de los temas fue lo de financiamiento" (Miembro de RUA).

Un ejemplo de esto es la eventual censura que tuvieron cuando quisieron dejar estipulado en el documento de cierre del CIDF, un párrafo alusivo a la crisis humanitaria que entonces estaban viviendo ciudadanos venezolanos varados en Tacna al no poder ingresar a Chile (García, 2019).

Como seres humanos y la cultura de paz nos preocupaba su situación (...) Decidimos tanto Arica como Tacna hacer un pronunciamiento, no partidario ni criticando a nadie, sino solicitando un lazo de humanidad y se nos negó, la respuesta fue de que no era la instancia de que no era correspondiente al comité este nos 
daban la pauta a que dentro del consejo si podíamos hacerlo, más nos aconsejaban que era preferible de que no, o sea, podíamos hacer pronunciamiento por medio del fan page del consejo binacional, pero nos recomendaban de que no era idóneo, pero sí por supuesto que podíamos trabajarlo como organizaciones independientes (anónimo).

Otro representante señaló que se trató de censura.

Fuimos censurados dentro de este comité, nos dijeron si quieren lo hacen afuera, porque es un tema muy delicado. Quedamos sorprendidos, realmente queríamos hacer algo con estas personas, y nos dijeron "estas cosas no se hablan en el comité, sáquenlo de su acta” (anónimo).

Estas limitaciones estructurales se sortean en parte cada vez que el COBIJUV se consolida como grupo hacia dentro y hacia afuera. Hacia dentro cuando existe conciencia de las relaciones jerárquicas entre estos jóvenes y la cancillería que los reconoce como subcomisión en un órgano bilateral. Hacia fuera cuando hacen uso de sus propias redes para vigorizar su activismo ciudadano en una sinergia que involucra institucionalidades de ambos países, redes de agrupaciones y las de los propios agentes.

\section{Me visto de diplomático para ayudar a mi región}

Cuando indagué por su participación en los CF y la experiencia personal de los miembros con quienes pude conversar, otra figura recurrente es la percepción de estos como espacios altamente institucionalizados, repletos de protocolos, normas explicitas y sutiles etiquetas:

Sí, conozco a los cónsules. Me cuesta de repente recordar sus nombres y muchos de ellos a mí me han dicho. Me ven mi actitud y saben que yo soy ultra informal y dicen "este chico tiene propulsión" como para liderar, y claro si quieren que yo lidere saquemos un poco la formalidad y trabajemos de otra forma. Los diplomáticos son cuadrados y si ellos me dicen "no hay que 


\section{0 / Diego Riquelme Gómez}

trabajar así" yo tampoco me voy a enojar con ellos porque bueno es su opinión no importa, trabajemos así (anónimo).

La resignación —o quizás adaptación— es aceptada por una visión práctica. Si en el complejo entramado de las cancillerías el rol que ocupan los jóvenes es la de ser este "aire renovador" mencionado por los cónsules, este ímpetu es limitado por constricciones propias de la lógica de razón de Estado. "Era un tema muy delicado, un tercer país no puede ser mencionado en un órgano bilateral" (miembro de cancillería) fue una máxima y respuesta univoca que obtuve de todos los miembros de cancillería con los que pude hablar respecto del veto sufrido por los jóvenes.

Pese a las constricciones, la participación en el comité permite la interacción y el intercambio de experiencias de activistas comprometidos con una identidad transfronteriza:

Lo más potente son las redes de intercambio cultural entre Chile y Perú. Ese lazo de amistad que se genera entre las organizaciones es súper fuerte, el intercambio de experiencias entre los jóvenes, lo que no sé yo lo saben ellos (anónimo).

Este sentimiento de identidad es compartido por todos los miembros que pude entrevistar. Si bien al interior existen matices respecto de la gestión, sobre todo respecto a las constricciones propias que impone su institucionalización, el COBIJUV y los espacios en los que se despliega son valorados en términos del activismo, toda vez que lo potencian al permitir el intercambio de saberes y experiencias. En estas instancias codearse con altos funcionarios de las cancillerías es una herramienta que aporta experiencia al despliegue como activistas:

Para mí es el pan de cada día porque como estoy dentro de esta gestión tan importante, yo estoy dentro de otras gestiones (...) para mí lo más importante es el centro cultural de mi barrio, prefiero estar ahí que con los cónsules. Eso es lo que más me llena, es como decir, queremos hacer una actividad grande para generar un impacto para ver si pueden cambiar las mentalidades de las 
personas, vale la pena entonces tener que disfrazarse de diplomático, me da lo mismo si hay monedas o no hay monedas, pero tenemos que hacer las cosas y al final poder generar un impacto latinoamericano (anónimo).

En tanto, su participación en el entramado institucional suple la necesidad de innovación en la política exterior de la cancillería tal como lo señalaron altos funcionarios. La colaboración en el COBIJUV y la participación de aquel dentro del CIDF permiten a estos jóvenes activistas establecer contactos, consolidar redes, movilizar recursos, tanto materiales como humanos, e internacionalizar el activismo propio con homólogos al otro lado de la frontera.

\section{Conclusiones}

En este artículo me he aproximado a la forma en que se desarrollan las prácticas de participación de los jóvenes agrupados en el COBIJUV, que incluyen, pero no se agotan, en la Subcomisión de Voluntariado Juvenil del CIDF Tacna-Arica. Mediante la explicitación de mi proceso de socialización e inmersión como investigador en este terruño diplomático particular, he recuperado las trayectorias de sus miembros y la manera en que convergen las trayectorias, valoraciones y relaciones de alteridad que emergen en aquellas prácticas. Además, he mencionado algunos alcances y limitaciones, así como eventuales estrategias y proyecciones para sortearlas, a partir de la visión de sus propios miembros.

A partir de los datos etnográficos expuestos en este documento y las preguntas señaladas puedo señalar que las prácticas de participación, en primer lugar, emergen como un arreglo institucional entre ambas cancillerías. Luego, las prácticas diplomáticas de los jóvenes desbordan la institucionalidad toda vez que sus miembros desarrollan procesos de alteridad e identificación tanto hacia dentro como hacia fuera del consejo, pero también entre aquí y allá, entre Tacna y Arica. Se trata de una organización conformada dentro de la arquitectura de los cuerpos diplomáticos, que al mismo tiempo adquiere autonomía de forma progresiva. 


\section{I Diego Riquelme Gómez}

Los alcances de esta actividad diplomática adquieren sentido en el cotidiano vivir de los habitantes ubicados en los márgenes de los Estados nacionales. Aparecen iniciativas de hermandad y buena vecindad como un hito fundacional, las que luego devienen en actividades diplomáticas y paradiplomáticas que permiten la convergencia del interés de los jóvenes a nivel bifronterizo y binacional. Las limitaciones tienen que ver con constricciones propias de la cancillería, que han sido abordadas, grosso modo, en los resultados.

Respecto de las dinámicas de alteridad es posible señalar que, si los comités de integración son vistos como espacios de innovación en la relación bilateral de estos países, la inclusión de los jóvenes va un paso más allá al incluir la pluralidad de actores en este dispositivo de gestión fronteriza. En aquel se despliegan arreglos institucionales que permiten la aparición de actores emergentes o invisibilizados en la diplomacia tradicional. Se abren instancias públicas como la política exterior, de suyo hermética, en clave de profundizar la democracia ante la oportunidad de acercar el activismo de los jóvenes al CIDF.

En tanto, el enfoque etnográfico permite visibilizar las relaciones de poder existentes, pero también las dinámicas de alteridad, es decir, de percepción mutua que se superponen entre el quehacer diplomático y el activismo de los jóvenes.

Los jóvenes ocupan y optimizan este espacio concedido, adaptándose a las constricciones propias de lógicas asociadas a la razón de Estado y a la canalización del interés nacional, propio de las cancillerías. El COBIJUV, a su vez, trasciende la institucionalidad y adquiere carácter propio y autonomía relativa respecto del CIDF. Mientras que el primero cumple el rol de "renovar aires" en el espacio del CIDF, dicha apertura permite que estos agentes ocupen espacios políticos que les permiten acercarse a la esquiva diplomacia. Sobre todo, les permite consolidar redes de intercambio, experiencias y saberes con sus pares al otro lado de la frontera, con quienes tienen en común el interés por la consolidación de una cultura de paz y buena vecindad aquí y allá. 


\section{Referencias}

Álvarez Torres, C. (2019). Comité de Integración y Desarrollo Fronterizo Perú-Chile: aproximaciones al mundo social y empresarial en torno a la frontera. Si Somos Americanos, 19(2), 49-67. https://doi.org/10.4067/ S0719-09482019000200049

Astroza, P. (2018). Las unidades subestatales y el mundo global. Desafíos para el Estado y la diplomacia actual. Diplomacia, (139), 69-72.

Bernal Meza, R. (2018). Paradiplomacia y regionalismo en situación de relaciones políticas en conflicto: el caso de Chile y Bolivia. Revista de Ciencia Política, 35(3), 20-29. https://doi.org/10.4067/S0718-090X2015000300007

Bernal, J., \& Rochetti, L. (2017). Evaluación del impacto de la gestión del "Comité de Integración y Desarrollo Fronterizo Perú-Chile" como entidad articuladora de la relación fronteriza. Iberoamerican Business Journal, 1(1), 4-28. https://doi.org/10.22451/3002.ibj2017.vol1.1.11001

Carrasco, E. (2014). Los jóvenes de Chile y Perú promueven hermandad y paz. La Estrella de Arica, 8-9.

Cheuk, K.-K. (2016). Everyday Diplomacy among Indian Traders in a Chinese Fabric Market. The Cambridge Journal of Anthropology, 34(2), 42-58. https://doi.org/10.3167/ca.2016.340204

Chile, Ministerio de Relaciones Exteriores. (s. f.) Cooperación internacional. Recuperado de https://chile.gob.cl/peru/relacion-bilateral/cooperacion-internacional

Chile, Ministerio de Relaciones Exteriores. (27 de novimbre de 2018). Con 163 compromisos concluye el II Gabinete Binacional Chile - Perú. Recuperado de https://minrel.gob.cl/minrel/noticias-anteriores/con-163-compromisos-concluye-el-ii-gabinete-binacional-chile-peru

Constantinou, C. M., Kerr, P., \& Sharp, P. (2016). Introduction. En Autores (Eds.), The SAGE Handbook of Diplomacy (pp. 1-11). SAGE Publications. Cornago, N. (2016). Diplomacia como heterología: pluralism social y multiples mediaciones institucionales en la frontera. En S. González, N. Cornago, \& C. Ovando (Eds.), Relaciones transfronterizas yparadiplomacia en América Latina: Aspectos teóricos y estudio de casos (pp. 17-45). Ril editores.

Diario Correo. (1 de marzo de 2016). Consejo Binacional de la Juventud busca la integración de Perú y Chile. Diario Correo, https:/ / diariocorreo.pe/edicion/tacna/consejo-binacional-de-la-juventud-busca-laintegracion-de-peru-y-chile-657181/?ref $=\mathrm{dcr}$ 
Dilla, H., \& Álvarez, C. (2019). La vuelta de todo eso. Economía y sociedad en la frontera chileno/peruana: el complejo urbano transfronterizo Tacna/Arica. Ril Editores

Durand, J. (2014). Coordenadas metodológicas. De cómo armar el rompecabezas. En C. Oehmichen Bazán (Ed.), La etnografía y el trabajo de campo en las ciencias sociales (pp. 261-284). UNAM.

García Espín, P. (2017). Etnografía y ciencia política: La excepcionalidad del caso español. Politica y Sociedad, 54(1), 255-275. https://doi. org/10.5209/POSO.48938

García, V. (14 de julio de 2019). La migración venezolana abre una pelea entre Perú y Chile: hay 1000 varados. La Nación. Recuperado de https:/ / www.lanacion.com.ar/el-mundo/la-migracion-venezolana-abre-unapelea-entre-peru-y-chile-hay-1000-varados-nid2267494/

Foucault, M. (2008). Seguridad, territorio, población (Vol. 265). Ediciones AKAL.

Geertz, C. (1991). La interpretación de las culturas. Descripción densa: hacia una teoría interpretativa de la cultura. Gedisa Editorial.

González, S., Cornago, N., \& Ovando, C. (2016). Relaciones transfronterizas y paradiplomacia en América Latina: Aspectos teóricos y estudio de casos S. González, N. Cornago, \& C. Ovando (Eds.). Ril editores.

Keating, M. (2001). Regiones y asuntos internacionales: motivos, oportunidades y estrategias. En F. Aldecoa, \& M. Keating (Eds.), Paradiplomacia: las relaciones internacionales de las regiones (pp. 11-28). Marcial Pons.

Kuus, M. (2019). The terroir of bureaucratic practice: Everyday life and scholarly method in the study of policy. Environment and Planning C: Politics and Space, 37(4), 617-633. https://doi.org/10.1177/0263774X18802954

Marcus, G. E. (2001). Etnografía en/del sistema mundo. El surgimiento de la etnografía multilocal. Alteridades, 11(22), 111-127. https://www. redalyc.org/pdf/747/74702209.pdf

Marsden, M., Ibañez-Tirado, D., \& Henig, D. (2016). Everyday diplomacy. The Cambridge Journal of Anthropology, 34(2), 2-22. https://doi. org/10.3167/ca.2016.340202

McConell , F., \& Dittmer, J. (2016). Diplomatic culture. En C. M. Constantinou, P. Kerr, \& P. Sharp, (Eds.), The SAGE Handbook of Diplomacy (104-113). SAGE Publications.

Mendoza, J. E. (2017). Razonamiento geopolitico. construcción de representaciones y códigos geopolíticos de Chile y sus vecinos. Sello editorial Universidad de Concepción. 
Milet, P. V. (2005). Chile-Perú: las raíces de una difícil relación. Estudios Internacionales (Santiago de Chile), 38(150), 59-73. https://doi. org/10.5354/0719-3769.2011.14385

Montsion, J. M. (2018). Ethnography and international relations: situating recent trends, debates and limitations from an interdisciplinary perspective. Journal of Chinese Sociology, 5(1). https://doi.org/10.1186/ s40711-018-0079-4

Neumann, I B. (2010). Autobiography, ontology, autoethnology. Review of International Studies, 36(4), 10511055. https://doi.org/10.1017/ S0260210510001191

Neumann, I. B. 2012a. At home with the diplomats: inside a European foreign ministry. Cornell University Press.

Neumann, I. B. 2012b. Diplomatic sites: A critical enquiry. Columbia University Press.

Oddone, N. (2018). Paradiplomacia Transfronteriza: la constitución de una comunidad epistémica latinoamericana. En K. Ramírez, \& A. Figueroa (Eds.), Gobernanza, sociedad civily frontera (pp. 15-47). Universidad Autónoma de Baja California.

Oglesby, D. (2016). Diplomatic langauge. En C. M. Constantinou, P. Kerr, \& P. Sharp, (Eds.), The SAGE Handbook of Diplomacy (242-254). SAGE Publications.

Ovando, C. (2017). Tacna y Arica en el marco del fallo de La Haya: algunas expresiones de integración desde la paradiplomacia y la sociedad civil. En P. Milet. (Ed.), Desafíos en la relación Chile-Perú (pp. 63-76). Santiago de Chile: Instituto de Estudios Internacionales de la Universidad de Chile, Instituto de Estudios Internacionales de la Pontificia Universidad Católica del Perú.

Ovando Santana, C., \& González Miranda, S. (2018). El papel de la paradiplomacia entre el norte de Chile y el sur del Perú: antecedentes históricos, limitaciones institucionales y nuevos desafíos "posfallo de La Haya”. Diálogo Andino, (55), 79-91. https://doi.org/10.4067/S071926812018000100079

Pajuelo, G. (2013). Evaluación del grado de cumplimiento de los acuerdos del comité de frontera Perú-Chile en su primera década de funcionamiento y perspectivas de la relación fronteriza. (Tesis de maestría, Universidad de Tarapacá, Chile). 


\section{6 / Diego Riquelme Gómez}

Riquelme, D. (2020). Mapa de actores que participan en los comités de frontera Perú-Chile. La emergencia de los jóvenes en la integración fronteriza. En Konrad Adenauer Stiftung —KAS— (Ed.), Premio 2019. Integración y Buena Vecindad. Chile-Perú / Perú-Chile (pp. 33-53). Autor.

Ruiz Muriel, M. C., \& Álvarez Velasco, S. (2019). Excluir para proteger: la "guerra" contra la trata y el tráfico de migrantes y las nuevas lógicas de control migratorio en Ecuador. Estudios Sociológicos, 37(111), 689-725. https://doi.org/10.24201/ES.2019V37N111.1686

Ruiz, R. (2018). Zonas extremas continentales y política exterior: un escenario estratégico e impostergable para Chile. Diplomacia, (139), 44-51

Vrasti, W. (2008). The strange case of ethnography and international relations. Millennium,37(2), 279-301. https://doi.org/10.1177/0305829808097641

Vrasti, W. (2010). Dr. Strangelove, or how I learned to stop worrying about methodology and love writing. Millennium, 39(1), 79-88. https://doi. org/10.1177/0305829810371017

Wacquant, L. (2005). Indicaciones sobre Pierre Bourdieu y la política democrática. En Autor (Coord.), El misterio del ministerio. Pierre Bourdien y la política democrática (pp. 23-42). Gedisa Editorial. 